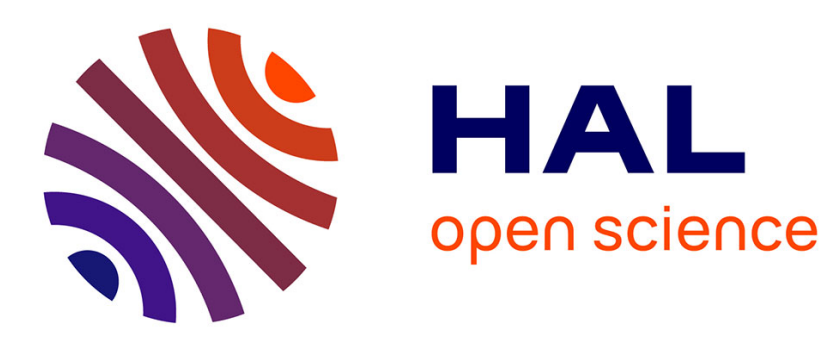

\title{
Étude des phénomènes prémonitoires des transitions de phases dans les alliages, par microscopie et diffraction électroniques
}

\author{
F. Reynaud
}

\section{- To cite this version:}

F. Reynaud. Étude des phénomènes prémonitoires des transitions de phases dans les alliages, par microscopie et diffraction électroniques. Revue de Physique Appliquée, 1980, 15 (2), pp.297-306. 10.1051/rphysap:01980001502029700 . jpa-00244729

HAL Id: jpa-00244729

https://hal.science/jpa-00244729

Submitted on 1 Jan 1980

HAL is a multi-disciplinary open access archive for the deposit and dissemination of scientific research documents, whether they are published or not. The documents may come from teaching and research institutions in France or abroad, or from public or private research centers.
L'archive ouverte pluridisciplinaire HAL, est destinée au dépôt et à la diffusion de documents scientifiques de niveau recherche, publiés ou non, émanant des établissements d'enseignement et de recherche français ou étrangers, des laboratoires publics ou privés. 


\title{
Étude des phénomènes prémonitoires des transitions de phases dans les alliages, par microscopie et diffraction électroniques $\left(^{*}\right)$
}

\author{
F. Reynaud \\ Laboratoire d'Optique Electronique, Laboratoire propre du C.N.R.S., associé à l'Université Paul-Sabatier, \\ 29, rue Jeanne-Marvig, B.P. 4347, 31055 Toulouse Cedex, France
}

\begin{abstract}
Résumé. - On passe en revue les phénomènes prémonitoires des transitions de phases qui sont visibles dans les alliages en microscopie et diffraction électroniques. Dans la première partie, on étudie les phénomènes prémonitoires des transitions de phases substitutionnelles (préprécipitation dans les solutions solides terminales, ordre à courte distance, fluctuations de composition dans le régime de décomposition spinodale, ...). La seconde partie est consacrée aux transitions de phases avec déplacement; on y fait le point sur le comportement prémartensitique des composés $\beta^{\prime}$ de Hume-Rothery.
\end{abstract}

\begin{abstract}
Phase transition premonitory phenomena are reviewed, which may be observed in alloys by electron microscopy and diffraction. In the first part, premonitory phenomena in substitutional solid solutions are analysed (preprecipitation, short range order, composition fluctuations in spinodal decomposition, ...). The second part is devoted to displacive transformations; in particular, the premartensitic behaviour of $\beta^{\prime}$ Hume-Rothery compounds is discussed.
\end{abstract}

1. Introduction. - Depuis une quinzaine d'années, la microscopie électronique s'est avérée une technique particulièrement bien adaptée à l'observation directe et à l'étude des domaines et des parois, dans le cas des transitions de phases [1], [2], [3] : domaines antiphasés et macles d'ordre, précipités, domaines ferromagnétiques et antiferromagnétiques, domaines ferroélectriques et antiferroélectriques, domaines ferroélastiques, ... Dans la plupart des systèmes simples, les principaux problèmes d'identification des domaines et de simulation de contraste sur les parois sont résolus, et les chercheurs commencent à s'intéresser aux phénomènes prémonitoires.

Ces phénomènes précèdent et annoncent les transitions de phases; ils existent dans la phase de basse température et dans la phase de haute température (même si l'on ne les a presque exclusivement étudiés que dans la phase de haute température), dans les transitions du premier et du second ordre (même s'ils sont différents au voisinage d'un point critique). Ils proviennent de l'existence de fluctuations macroscopiques du paramètre d'ordre $\langle\eta\rangle$. Ces fluctuations sont caractérisées par la variance $\left\langle\Delta \eta^{2}\right\rangle$ de la variable microscopique dont le paramètre d'ordre à longue distance est la moyenne. Cette variance, que l'on appelle fonction de corrélation des fluctuations, est

$\left(^{*}\right)$ Conférence présentée au Congrès de la Société Française de Physique (Toulouse). proportionnelle à la susceptibilité généralisée, d'après le théorème de la fluctuation et de la dissipation [4]. Nous avons représenté schématiquement sur la figure 1 les variations de $\langle\eta\rangle$ et de $\left\langle\Delta \eta^{2}\right\rangle$, en fonction de la température, dans le cas d'une transition du second ordre.

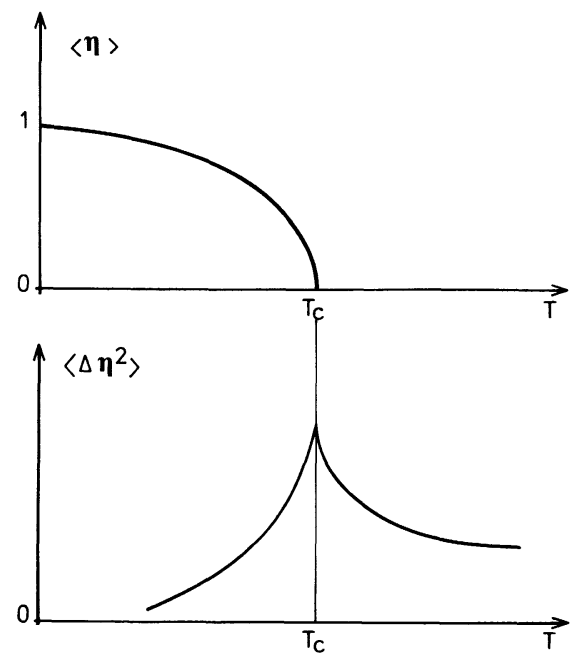

Fig. 1. - Variation schématique du paramètre d'ordre à longue distance $\langle\eta\rangle$ et de la fonction de corrélation des fluctuations $\left\langle\Delta \eta^{2}\right\rangle$ en fonction de la température, dans le cas d'une transition du second ordre.

[Schematic variation of the long-range order parameter $\langle\eta\rangle$ and the correlation function of the fluctuations $\left\langle\Delta \eta^{2}\right\rangle$ with temperature, for a second order transition.] 
Les phénomènes prémonitoires des transitions de phases se manifestent par l'existence d'anomalies dans les propriétés physiques des matériaux : résistivité électrique, chaleur spécifique, constantes élastiques, ... Nous nous limiterons toutefois dans cet article aux effets prémonitoires observés dans les alliages métalliques, en diffraction et microscopie électroniques; il s'agit essentiellement de traînées diffuses ou de maximums d'intensité diffuse dans les diagrammes de diffraction, et d'anomalies du contraste dans les micrographies (structure de tweed, peau d'orange, ...). Nous insisterons surtout sur les effets visibles sur les diagrammes de diffraction, car ce sont généralement ceux qui fournissent le plus d'informations, Il n'existe pas d'article de revue complet sur ce sujet, mais plusieurs exposés récents [5], [6], [7] traitent chacun un aspect de ce thème.

Nous distinguerons, comme on le fait habituellement, le cas des transitions substitutionnelles (précipitation cohérente, décomposition spinodale, ordredésordre, ordre des lacunes, ...) et celui des transitions avec déplacement (transitions martensitiques, ferroélastiques, ...).

2. Les transitions de phases substitutionnelles. Nous parlerons dans ce paragraphe des transitions de phases dans lesquelles le phénomène principal est la répartition des différents types d'atomes sur le réseau moyen d'une solution solide substitutionnelle (avec intervention obligatoire de la diffusion), les éventuels déplacementsdes atomes par rapport au réseau moyen ne constituant qu'un effet secondaire. Selon le signe de

$$
\frac{1}{2}\left(V_{\mathrm{AA}}+V_{\mathbf{B B}}\right)-V_{\mathrm{AB}},
$$

les solutions solides ont tendance à l'ordre ou à la ségrégation, mais nous insisterons ici surtout sur l'ordre à courte distance $(\S 2.1)$, car les phénomènes prémonitoires sont mieux documentés dans le cas de la ségrégation (zones G.P. et fluctuations de composition en régime spinodal, $\S 2.2$ ).

2.1 LES PHÉNOMÈNES PRÉMONITOIRES DANS LES SOLUTIONS SOLIDES PRÉSENTANT UNE TENDANCE A L'ORDRE : L'ORDRE A COURTE DISTANCE. - La compréhension de l'ordre à courte distance [6], [7], phénomène prémonitoire de l'ordre à longue distance, a été récemment influencée par les progrès concernant l'ordre à longue distance lui-même.

Les premiers concernent la description des structures ordonnées à longue distance à l'aide d'ondes statiques de concentration [8]. Ce mode de description est utile pour l'exploitation des diagrammes de diffraction et pour la physique statistique classique de la transition ordre-désordre [8]; d'autre part, il précise l'analogie avec les ondes de spin en magnétisme et les modes mous dans les transitions de phases avec déplacement : la transition désordre-ordre apparait comme la condensation statique d'ondes dynamiques de concentration.
D'autres progrès consistent en la certitude que les transitions $\mathrm{A} 2 \rightarrow \mathrm{B} 2$ et $\mathrm{B} 2 \rightarrow \mathrm{DO}_{3}$ sont du second ordre. Après une longue bataille à laquelle ont participé les microscopistes [9], [10], [11], cette certitude a été définitivement acquise par la mise en évidence expérimentale et l'étude quantitative du comportement critique de $\mathrm{CuZn}$ [12-17], $\mathrm{FeCo}$ [18], $\mathrm{Fe}_{3} \mathrm{Al}$ [9], [19], [20]... Il est intéressant de remarquer que les transitions du second ordre $\mathrm{A} 2 \rightarrow \mathrm{B} 2$ et $\mathrm{B} 2 \rightarrow \mathrm{DO}_{3}$ ne sont pas frustrantes [21], c'est-à-dire que la structure ordonnée considérée n'impose pas de mauvaises paires A-A ou B-B (contrairement, par exemple, à la transition frustrante $\mathrm{A} 1 \rightarrow \mathrm{L1}_{2}$ ).

2.1.1 Relation entre l'intensité diffuse et la surface de Fermi. - L'ordre atomique modifie la surface de Fermi des alliages (ne serait-ce que par l'effet géométrique de l'augmentation du volume de la maille primitive). Cet effet a été signalé tout d'abord dans les alliages à longue période [22]. En fait, les maximums d'intensité diffuse pourraient être considérés comme une image de la surface de Fermi [23], [24], [25].

2.1.2 Microscopie électronique de l'ordre à courte distance. - On a utilisé la microscopie électronique pour essayer de résoudre la querelle entre l'ordre à courte distance homogène et les microdomaines, soit en sélectionnant avec le diaphragme objectif une tache diffuse d'ordre à courte distance, soit en utilisant la haute résolution [26]. On a ainsi étudié les systèmes $\mathrm{Cu}-\mathrm{Pt}$ [27], [28], $\mathrm{Cu}-\mathrm{Al}$ [29], $\mathrm{Cu}-\mathrm{Pd}$ [28], Ni-Mo [30], ainsi que d'autres systèmes [31], [32]. La querelle [33] des microdomaines rappelle celle des zones G.P., lorsqu'on se posait la question de savoir s'il s'agissait d'une véritable phase. Une onde de concentration dynamique trempée depuis $T>T_{\mathrm{c}}$ ne constitue pas une phase ordonnée, puisqu'elle n'admet pas de limite bien définie et qu'elle varie de manière continue. Toutefois, elle doit donner un effet de facteur de structure et donc être visible dans les micrographies en champ sombre.

2.1.3 La méthode de Ridder. - Généralisant une méthode [34] utilisée en diffraction de rayons $X$, de Ridder et col. [35], [36] proposent de décrire les états donnant lieu à des traînées diffuses par des amas dont la nature est déduite du développement en série de Fourier du lieu géométrique idéalisé des maximums d'intensité diffuse [37], [38], [39]; nous en montrons un exemple d'application à la figure 2 . Bien adaptée aux problèmes d'ordres unidimensionnel et bidimensionnel, cette méthode présente l'avantage d'être la seule technique d'exploitation quantitative des traînées diffuses dans le cas de la diffraction électronique. Elle ne me paraît toutefois pas être de nature à résoudre le problème de fond que pose la représentation classique de l'ordre à courte distance dans les alliages.

2.1.4 Le problème de la représentation de l'ordre à courte distance. - On sait depuis longtemps que 


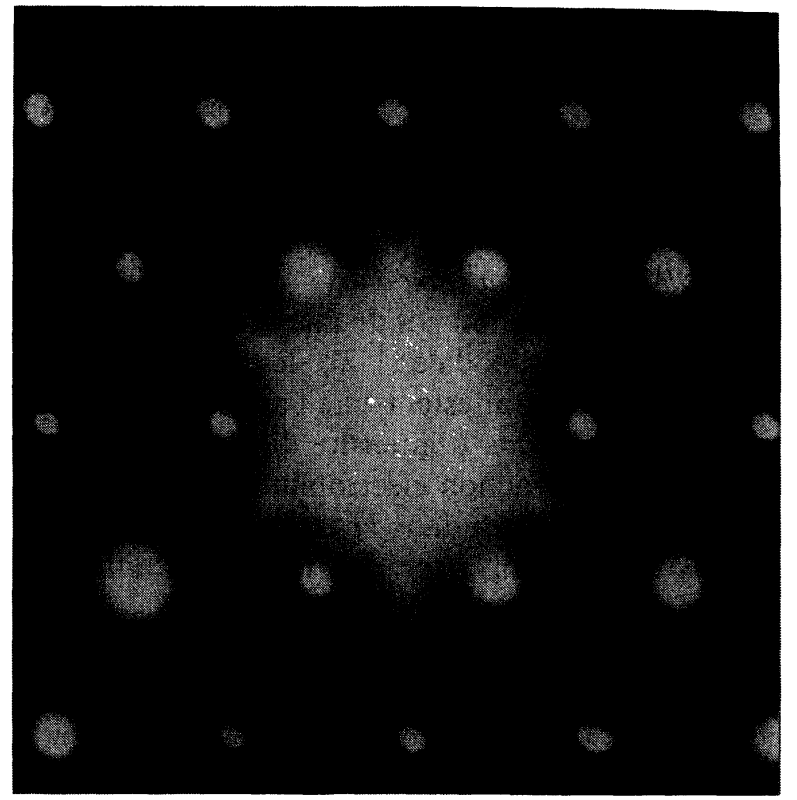

a)
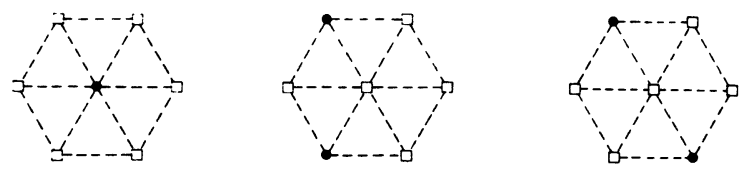

b)
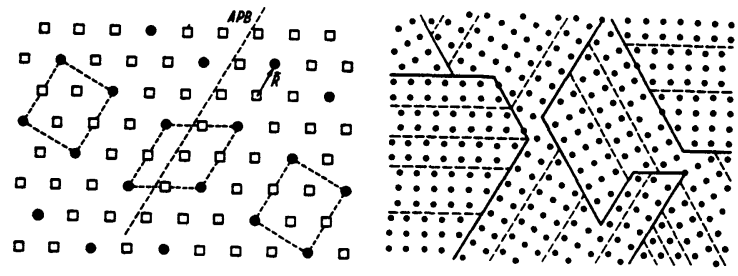

c)

Fig. 2. - Exemple d'application de la méthode de Ridder [35], [36] :a) intensité diffuse dans $\mathrm{Cu}_{0,50} \mathrm{NbS}_{2} ; b$ ) les principaux amas correspondants; $c$ ) exemple de modèle déduit de la figure $2 b$.

[Application of de Ridder's method (by courtesy of [35], [36]) : a) diffuse intensity in $\mathrm{Cu}_{0.50} \mathrm{NbS}_{2} ; b$ ) preponderant clusters; c) microdomains model.]

l'on ne peut pas représenter une solution solide présentant de l'ordre à courte distance par une maille élémentaire. Les deux seules approches classiques dont on dispose sont :

- Soit la donnée d'une ou de plusieurs configurations de l'alliage, en précisant l'occupation de chacun des $N$ sites du cristal (ou d'un grand nombre de sites). Toutefois, le nombre de configurations possibles peut être considérable, de sorte que celles que l'on a choisies peuvent ne pas être représentatives de l'alliage. D'autre part, on choisit généralement des configurations compatibles avec les paramètres macroscopiques d'ordres à courte et longue distances; on exclut ainsi des configurations qui sont peut-être peu probables, mais que l'on doit tout de même prendre en considération (la seule contrainte macroscopique dont on ne peut pas s'affranchir étant la composition de l'alliage).

- Soit la donnée des paramètres d'ordre à courte distance, déterminés à partir des mesures d'intensité diffuse en diffraction de rayons $\mathbf{X}$ ou de neutrons. Ces paramètres représentent toute l'information que l'on peut fournir sur la solution solide; ils représentent une moyenne, et ils ne peuvent pas être représentés de manière classique (sauf peut-être en donnant un rayon moyen, comme on le fait pour les zones G.P.), sous peine de contradictions; c'est ainsi que, d'après les probabilités composées, on pourrait déduire les paramètres d'ordre à courte distance entre voisins d'ordre supérieur du paramètre d'ordre à courte distance entre premiers voisins.

Les contradictions auxquelles se heurte la conception classique de l'ordre à courte distance devraient être résolues dans un futur proche par l'introduction d'une description quantique.

En attendant, lorsqu'on a des maximums d'intensité diffuse dont l'origine présumée est l'ordre à courte distance (Fig. 3a), on essaie d'obtenir par recuit la formation de l'ordre à longue distance correspondant (Fig. 3b). Lorsque l'on a affaire à un problème purement substitutionnel, les diffractions électroniques permettent dans certains cas de déterminer la surstructure. Dans l'exemple de la figure 3, on a pu déterminer sans ambiguité par diffraction électronique seulement la surstructure $\mathrm{Ni}_{2} \mathrm{Al}$ ([40], Figs. $3 c$ et $3 d$ ).

2.2 LES PHÉNOMÈNES PRÉMONITOIRES DANS LES SOLUTIONS SOLIDES PRÉSENTANT UNE TENDANCE A LA SÉGRÉGATION : LES ZONES G.P. ET LES FLUCTUATIONS SPINODALES. - Les phénomènes prémonitoires sont différents selon que l'on a affaire à la précipitation dans une solution solide terminale ou à la décomposition spinodale d'un alliage concentré.

2.2.1 La préprécipitation dans les solutions solides terminales; les zones de Guinier et Preston (G. P.). Les phénomènes de préprécipitation dans les solutions solides terminales ont surtout été étudiés par diffusion centrale de rayons X [41]. Dans le cas de la diffusion des électrons, la présence de zones G. P. se manifeste :

- Dans les diagrammes de diffraction par des traînées diffuses, auxquelles contribuent [42] l'effet de forme des zones, l'effet de distorsion élastique de la matrice et un éventuel effet de facteur de structure. Lors d'un épaississement de zones G. P. planes, on passe ainsi progressivement de traînées continues à des maximums d'intensité diffuse.

- Dans les micrographies où on les observe alors directement en champ clair [43], [44], [45], en champ sombre sur les maximums d'intensité diffuse [44] et en champ sombre sur une tache faiblement excitée de la matrice [46], [47], [48]. C'est cependant la microscopie 


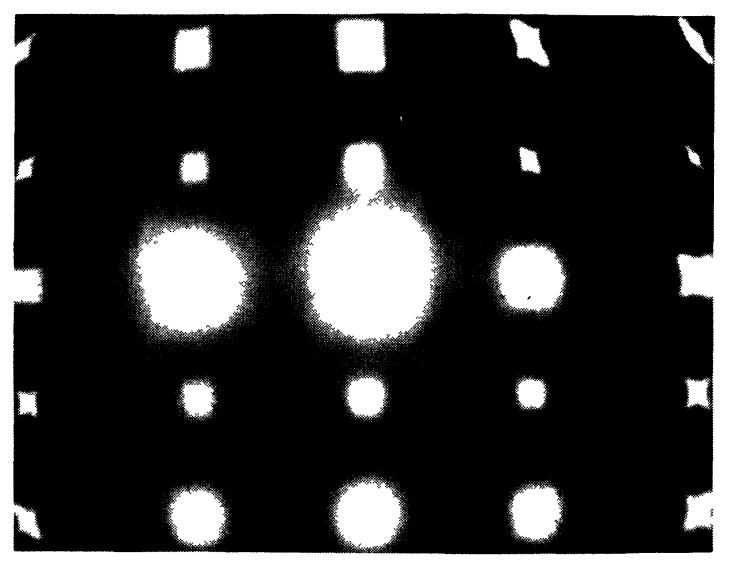

a)

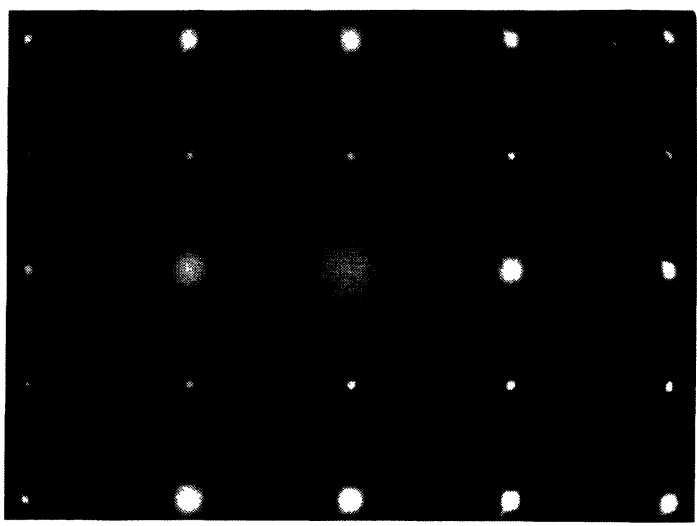

b)

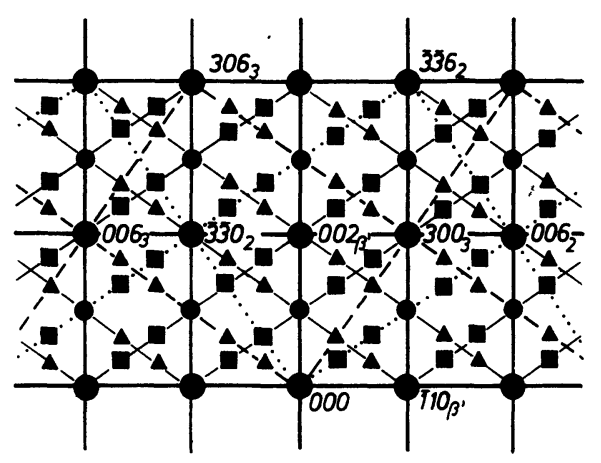

c)

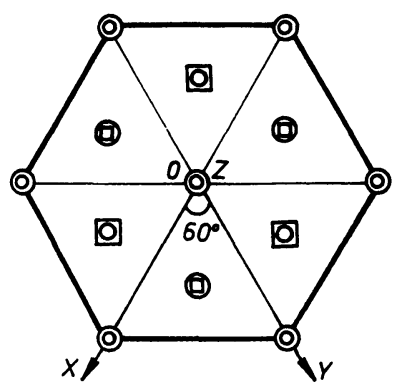

(1) Ni $Z=0,1 / 2,1$

(1) $\begin{cases}\text { Al } & Z=1 / 6 \\ \text { Ni } & Z=2 / 3\end{cases}$

O $\begin{cases}\text { Ni } & Z=1 / 3 \\ \text { Al } & Z=\frac{5}{6}\end{cases}$

d)

Fig. 3. - Diagramme de diffraction $\{110\}^{*}$ dans Al62,5 at \% $\mathrm{Ni}[40]$. a) Maximums d'intensité diffuse dus à l'ordre à courte distance entre atomes de nickel d'anti-structure ; $b$ ) taches de surstructure dues à l'ordre à longue distance $\mathrm{Ni}_{2} \mathrm{Al} ; c$ ) indexation correspondante; $d$ ) maille élémentaire de la surstructure $\mathrm{Ni}_{2} \mathrm{Al}$.

$\left[\{110\}^{*}\right.$ diffraction pattern in Al-62.5 at \% Ni [40]. a) Diffuse intensity due to short-range ordering of nickel antistructure atoms; $b$ ) long-range order reflections of the superstructure $\mathrm{Ni}_{2} \mathrm{Al}$;

c) indexation of figure $3 b ; d$ ) unit cell of $\mathrm{Ni}_{2} \mathrm{Al}$.]

électronique à haute résolution qui apporte l'information la plus fine [43], [44], [49]; la visualisation directe plans réticulaires permet en effet de montrer que, très petit nombre de couches atomiques [50], [51].

2.2.2 L'apparition des fluctuations de composition, dans la décomposition spinodale. - Lors de la décomposition d'une solution solide par le mécanisme de la décomposition spinodale [52], les fluctuations de composition se manifestent par l'apparition et l'évolution de maximums d'intensité diffuse au voisinage des taches de diffraction électronique. Ces maximums sont en général noyés dans la tache centrale, mais ils sont visibles au voisinage des taches de diffraction
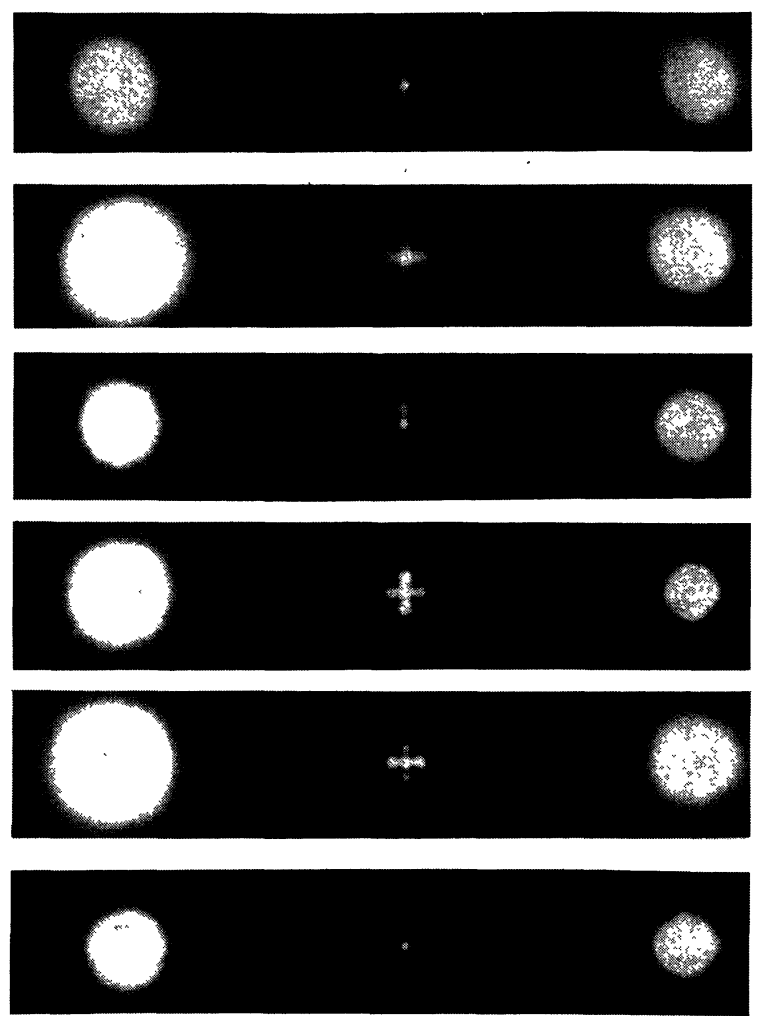

Fig. 4. - Evolution en fonction de la durée (en min) de recuit à $200^{\circ} \mathrm{C}$ de l'intensité diffuse au voisinage d'une tache de surstructure, lors de la décomposition spinodale d'un alliage $\mathrm{Cu}_{2,5} \mathrm{Mn}_{0,5} \mathrm{Al}$

[Diffuse intensity around a superlattice reflection as a function of aging time (indicated in minutes) at $200{ }^{\circ} \mathrm{C}$, in the case of spinodal decomposition of an $\mathrm{Cu}_{2.5} \mathrm{Mn}_{0.5} \mathrm{Al}$ alloy (by courtesy of [58]).] 
d'ordre élevé dans Au-Ni [53], Ni-Ti [54], Cu-Ni$\mathrm{Fe}[55] \ldots$, et au voisinage des réflexions de surstructure, lors de la décomposition d'un alliage Ni33,6 at $\% \mathrm{Mn}$ en les phases $\mathrm{L}_{2}$ et $\mathrm{L}_{1}$ [56], [57] et des alliages $(\mathrm{Cu}-\mathrm{Mn})_{3} \mathrm{Al}$ en les phases $\mathrm{L}_{1}$ et $\mathrm{DO}_{3}(58)$ (Fig. 4). La décomposition spinodale se manifeste dans les micrographies électroniques par des effets de contraste dus aux variations de paramètre cristallin associées aux fluctuations de composition [56], [57], [58].

Considéré d'abord comme une curiosité dans les alliages ordonnés, il semble que le mécanisme de décomposition spinodale [59] y soit très courant; on vient même de mettre en évidence récemment la décomposition spinodale du laiton $\beta^{\prime}$ [60]. Il n'y a pas là de contradiction, car il s'agit, dans les cas cités, d'un ordre entre premiers voisins et d'une démixtion selon le mécanisme spinodal entre seconds voisins. Signalons enfin que le mécanisme de la décomposition spinodale est fréquent dans les minéraux [61].

3. Les transitions de phases avec déplacement : le comportement "prémartensitique " des composés $\beta^{\prime}$ de Hume-Rothery. - Depuis une vingtaine d'années, le concept de mode mou introduit en théorie des transitions de phases avec déplacement par Cochran [62], [63] s'est avéré particulièrement fructueux. Dans sa présentation la plus simple, un mode mou est un mode normal de vibration du cristal (phonon) dont l'amplitude augmente et dont la fréquence tend vers zéro lorsqu'on s'approche du point de transition. Il y a alors condensation du mode de vibration, et la structure de la phase de basse température est déterminée par celle de la phase de haute température et par le vecteur propre du mode mou. Cette notion fondamentale montre que l'on passe de manière continue (au moins dans le cas d'une transition du second ordre) d'un phénomène dynamique à un phénomène statique. Ce schéma simpliste est en général compliqué par des effets d'anharmonicité et d'interaction entre modes; il correspond toutefois assez bien à la réalité dans les pérovskites ou les $\mathrm{A} 15$, par exemple. Nous signalons deux articles de revue [64], [65] et un livre [66] récents sur les modes mous.

3.1 Les Composés $\beta^{\prime}$ DE Hume-RotherY. - Les métallurgistes ont alors essayé d'appliquer la notion d'instabilité d'un cristal par rapport à un mode de vibration à la transformation martensitique des composés $\beta^{\prime}$ de Hume-Rothery [67], [68], [69]. Ces composés présentent la structure $\mathrm{A} 2, \mathrm{~B} 2, \mathrm{DO}_{3}$ ou $\mathrm{L} 2{ }_{1}$. Ils correspondent à une concentration électronique égale à $3 / 2 e / a$. Enfin, on sait depuis Zener que le réseau c.c. est intrinsèquement peu résistant à un cisaillement $\langle 110\rangle\{1 \overline{1} 0\}$. Nous $n$ 'insisterons pas sur la faiblesse de la constante de cisaillement correspondante $C^{\prime}$ et sur son adoucissement $\left(\mathrm{d} C^{\prime} / \mathrm{d} T>0\right)$, et nous renvoyons le lecteur à deux articles de revue récents [70], [71]. Les métallurgistes ont très vite buté sur une difficulté, à savoir que, contrairement au cas des A15 par exemple, la transformation martensitique se produisait avant que $C^{\prime}$ ne s'annule. On a donc été amené à penser que la transformation martensitique se produisait préférentiellement sur des hétérogénéités [72], [73]. Or, la microscopie et la diffraction électroniques ont effectivement montré que les hétérogénéités ne manquaient pas dans les composés $\beta^{\prime} \ldots$ Nous allons nous efforcer, dans ce qui suit, de dégager les idées qui semblent s'imposer depuis le dernier article de revue sur ce sujet [74].

3.2 LES PRÉPRÉCIPITÉS OMÉGA DANS LES COMPOSÉS De HuMe-Rothery a BASE DE $\mathrm{Cu}, \mathrm{Ag}$ et $\mathrm{Au}$. - Les premières anomalies dans les diagrammes de diffraction électronique ont été observées dans TiNi [75], [76], [77] : plans $\{111\}^{*}$ d'intensité diffuse et taches de diffraction inattendues. Les explications proposées faisaient intervenir une distorsion rhomboédrique [75], [77], une phase intermédiaire entre la phase B2 et la martensite [78], [79], [80], ainsi que les phases proches $\mathrm{NiTi}_{2}$ et $\mathrm{Ni}_{3} \mathrm{Ti}[79]$.

Dans le système $\mathrm{Cu}-\mathrm{Sn}$ on avait observé à haute température dans le microscope électronique [81] des traînées dans les directions $\langle 110\rangle^{*},\langle 112\rangle^{*}$ et $\langle 123\rangle^{*}$, correspondant également à des plans $\{111\}^{*}$ d'intensité diffuse. Les travaux les plus importants restent ceux de Deruyttère et col. [82], [83] ; certaines anomalies sont attribuées à une phase bainitique $\alpha^{\prime}$ [82], d'autres à une phase $\delta^{\prime}$ proche de la phase $\mathrm{Cu}-\mathrm{Sn} \delta$ [83]; d'autres taches enfin, qui avaient également été observées par Warlimont [84], sont attribuées à la précipitation d'une phase oméga analogue à celle des alliages de $\mathrm{Ti}, \mathrm{Zr}$ et $\mathrm{Hf}$ [83].

Parmi les anomalies observées en diffraction électronique dans les systèmes $\mathrm{Ag}-\mathrm{Zn}$ [85] et $\mathrm{Ag}-\mathrm{Mg}$ [86], il faut signaler la présence de réflexions intenses, de forme ellipsoïdale, situées à $1 / 3$ et $2 / 3$ des taches 112 et légèrement décalées par rapport à la position idéale, dans les diagrammes $\{110\}^{*} ;$ l'intensité de ces taches correspond au facteur de structure de la maille oméga des alliages de titane, et la similitude a été signalée par les auteurs de [85] et [86].

C'est cependant dans le laiton $\beta^{\prime}$ et dans les alliages ternaires dérivés que la controverse a été la plus développée; en 1968 en effet, Sato et Takezawa [87] présentaient un diagramme $\{110\}^{*}$ sur lequel on observait des traînées $\langle 112\rangle^{*}$ et des réflexions ellipsoïdales inattendues (voir Fig. 5a). Ces auteurs concluaient à l'existence de précipités cohérents; ils observaient également des traînées diffuses liées à la martensite. En 1971, J. Van Paemel [88] montrait que les réflexions observées par [87] pouvaient s'expliquer par l'existence de précipités de type oméga, alors que Sato et Takezawa [89] proposaient en 1973 une structure orthorhombique concurrente. Prasetyo et col. [90] confirmaient en 1976 la structure oméga et présentaient des images en champ sombre (éven- 
tuellement faiblement excité) des précipités allongés selon les directions $\langle 111\rangle$ (Fig. $5 b$ et $5 c$ ). Bien que l'on ait encore fait appel à la structure de Sato et Takezawa dans le système ternaire $\mathrm{Cu}-\mathrm{Zn}-\mathrm{Al}$ [91], il semble que l'on doive l'écarter : d'une part, elle

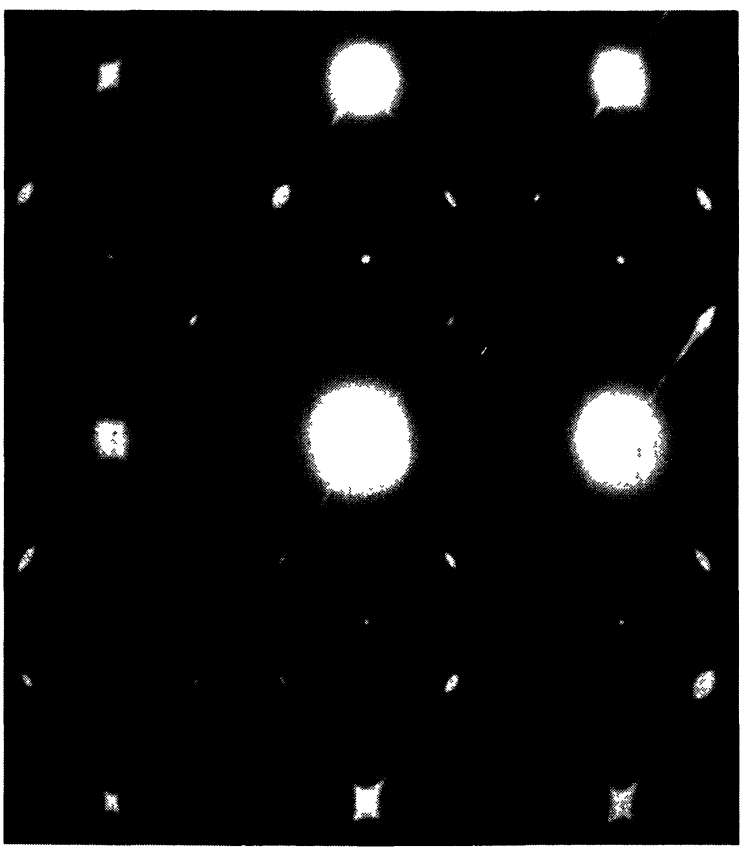

a)

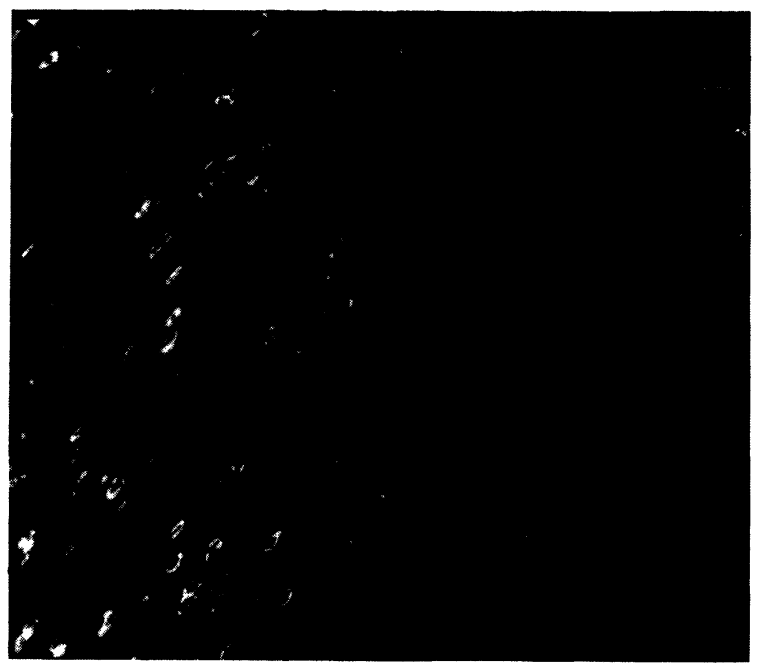

b)

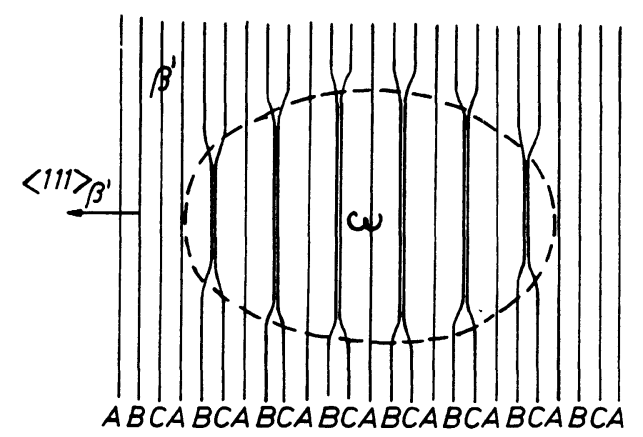

c) donnerait lieu à douze variantes (au lieu de quatre observées), d'autre part, elle a une densité qui est égale aux $2 / 3$ de celle de la matrice. La structure oméga a été d'ailleurs confirmée dans le laiton $\beta^{\prime}$ par [92].

Les mêmes précipités oméga ont été observés dans les alliages $\mathrm{Cu}_{2+x} \mathrm{Mn}_{1-x} \mathrm{Al}$, de structure $\mathrm{DO}_{3}$ [93], dans lesquels on a également suivi la formation d'une autre phase intermédiaire en microscopie électronique à basse température [94]. Les mêmes phénomènes sont observés dans les alliages ternaires $\mathrm{Cu}-\mathrm{Al}-\mathrm{Ni}$ (Fig. 6) [95], ainsi que dans $\mathrm{Cu}-$ $\mathrm{Zn}-\mathrm{Si}[96]$.

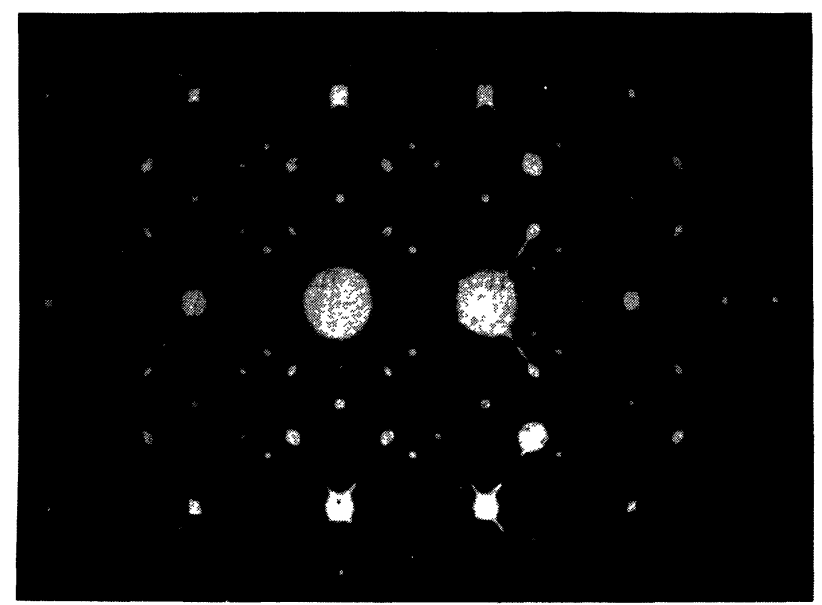

Fig. 6. - Diagramme de diffraction $\{113\}^{*}$ dans $\mathrm{Cu}-14 \%$ Al$4 \% \mathrm{Ni}$ (en poids) recuit une heure à $950^{\circ} \mathrm{C}$ et trempé à l'eau (cliché G. Hausch).

[\{113 $\}^{*}$ diffraction pattern in $\mathrm{Cu}-14 \% \mathrm{Al}-4 \% \mathrm{Ni}$ (weight \%) annealed one hour at $950{ }^{\circ} \mathrm{C}$ and water quenched (by courtesy of G. Hausch).]

Dans d'autres systèmes, par contre, on n'a obtenu ni précipités ni réflexion oméga, mais des phénomènes analogues à ceux que l'on appelle intensité oméga diffuse dans les alliages de $\mathrm{Ti}, \mathrm{Hf}$ ou $\mathrm{Zr}$, c'est-à-dire les phénomènes prémonitoires de la formation de la phase oméga. Lorsque les déplacements des rangées $\langle 111$ > sont couplés, comme dans Ag-Al [97], les phénomènes sont analogues à ceux que l'on observe, par exemple, dans les alliages $\mathrm{Ti}-11 \% \mathrm{Fe}$ (en poids) [98]. Dans d'autres systèmes, les traînées et les maximums d'intensité diffuse sont analogues à ceux que l'on observe dans le système $\mathrm{Zr}-\mathrm{Nb}$, pour des

Fig. 5. - Les précipités oméga dans le laiton $\beta^{\prime}(90)$. $a$ ) Diagramme de diffraction $\{110\}^{*}$ dans l'alliage $\mathrm{Cu}-46,8$ at $\% \mathrm{Zn}$ : on remarque des taches ellipsoïdales à $1 / 3$ et $2 / 3$ des taches principales; $b$ ) Micrographie électronique en champ sombre oméga faiblement excité dans l'alliage $\mathrm{Cu}-46,4$ at $\% \mathrm{Zn} ; c$ ) Schéma d'un préprécipité oméga.

[Omega preprecipitates in $\beta^{\prime}$-brass (90). a) $\{110\}^{*}$ diffraction pattern in $\mathrm{Cu}-46.8$ at \% $\mathrm{Zn}$. Ellipsoidal omega spots are observed at $1 / 3$ and $2 / 3$ of the matrix spots; $b$ ) Week-beam micrograph with an omega reflection, in $\mathrm{Cu}-46.4$ at $\% \mathrm{Zn} ; c$ ) Schematic omega preprecipitate.] 
compositions où la phase oméga ne se forme plus [99]; c'est le cas de Fe-Al [100] (Fig. 7) et de Co-Ga [101], [102] par exemple. Dans ces systèmes, comme peutêtre dans $\mathrm{Ni}-\mathrm{Al}$, on pourrait relier ces observations à une relaxation autour des lacunes [100-104]. Nous verrons plus loin que l'hypothèse d'un mode mou localisé permettrait de passer d'un déplacement dynamique à une relaxation statique.

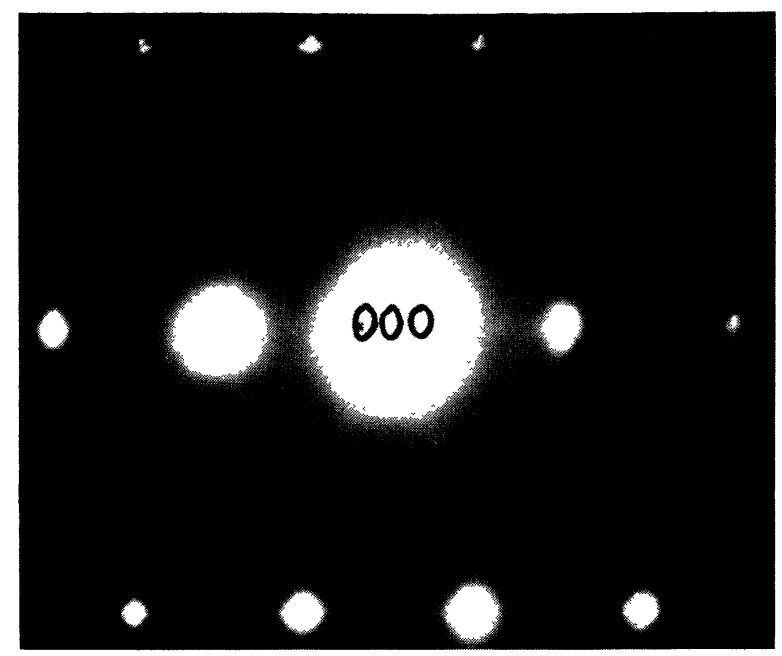

Fig. 7. - Diagramme de diffraction $\{113\}^{*}$ dans Fe-49 at \% $\mathrm{Al}$ (d'après [100]).

[\{113 $\}^{*}$ diffraction pattern in $\mathrm{Fe}-49$ at \% $\mathrm{Al}$ (by courtesy of [100]).]

On sait maintenant avec certitude que l'on observe, dans de nombreux composés $\beta^{\prime}$ de Hume-Rothery à base de $\mathrm{Cu}, \mathrm{Ag}$ et $\mathrm{Au}$, des phénomènes prémonitoires de la formation d'une structure oméga ou même de véritables précipités statiques, selon les systèmes. L'analogie avec les zones G. P., qui avait été soulignée par Ehemann [105] « (it seems to be appropriate to view omega as a form of G. P. zone characterized by displacement disorder) " et reprise indépendamment par [90], semble s'imposer. Ces préprécipités cohérents se forment par déplacement d'atomes au cours de la trempe, sans intervention de la diffusion. Au cours d'un recuit, la diffusion peut intervenir et la composition des préprécipités peut évoluer (ces phénomènes sont connus depuis longtemps dans les alliages à base de $\mathrm{Ti}, \mathrm{Hf}$ et $\mathrm{Zr}$ ). Les préprécipités oméga seraient donc la première structure intermédiaire vers la formation de la phase compacte voisine $\alpha$ (h.c. dans les alliages de $\mathrm{Ti}$, $\mathrm{Hf}$ et $\mathrm{Zr}$, c.f.c. dans les alliages des métaux nobles) dans la gamme des températures bainitiques [106], [107], méritant ainsi la dénomination de préprécipités. Ils ne constituent par contre vraisemblablement pas une structure intermédiaire obligatoire entre la matrice et la martensite à basse température; la formation de la structure oméga ne semble d'ailleurs pas liée directement à l'adoucissement de la constante $C^{\prime}$, qui correspond à un phonon de centre de zone, mais plutôt à l'anomalie observée au voisinage de $q=2 / 3|\xi \xi 0|$ dans la branche TA2 de $\mathrm{AuCuZn_{2 }}$ [108] et $\mathrm{Cu}-\mathrm{Zn}-\mathrm{Al}$ [109].

Dans le cas de l'intensité oméga diffuse, l'hypothèse d'un mode de vibration localisé [110], [111] (pouvant éventuellement devenir mou) sur les défauts ponctuels (essentiellement les lacunes, et peut-être les atomes d'anti-structure) permettrait de relier les observations aux modèles de relaxation autour des lacunes [100-104]. Cette hypothèse permettrait, dans les systèmes considérés, de faire la relation avec les anomalies de la diffusion, comme on vient récemment de le faire dans les cas des alliages à base de $\mathrm{Ti}, \mathrm{Hf}$ et $\mathrm{Zr}$ [112]. On peut en effet remarquer que les atomes qui quittent leur position pour se placer en position oméga créent ainsi des lacunes constitutionnelles dans la matrice.

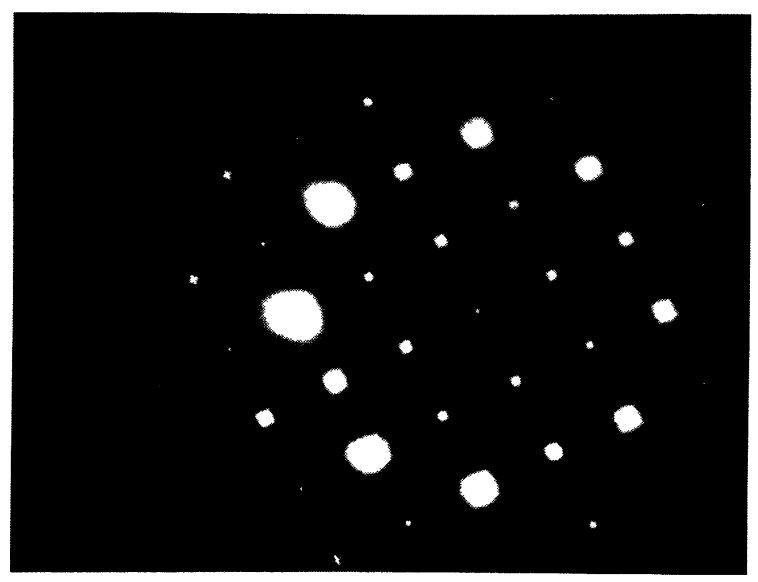

a)

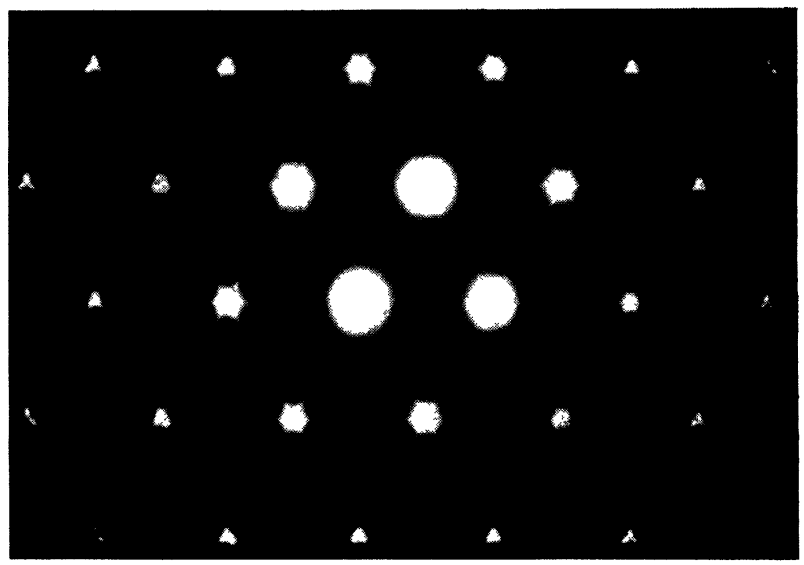

b)

Fig. 8. - Les traînées diffuses $\langle 110\rangle^{*}$ dans Ni-37,5 at \% Al. a) Diagramme de diffraction $\{100\}^{*}$ : deux familles de traînées sont situées dans le plan du diagramme, alors que les quatre autres sont également inclinées par rapport à ce même plan.b) Diagramme de diffraction $\{111\}^{*}$ : trois familles de traînées sont situées dans le plan du diagramme, alors que les trois autres sont également inclinées par rapport à ce même plan.

$\left[\langle 110\rangle^{*}\right.$ diffuse streaks in Ni-37.5 at \% Al. a) $\{100\}^{*}$ diffraction pattern : two families of streaks lie in the plane of the pattern, and the four others are steeply and equally inclined with respect to this plane. $b)\{111\}^{*}$ diffraction pattern : three families of streaks lie in the plane of the pattern, and the three others are steeply and equally inclined with respect to this plane.] 

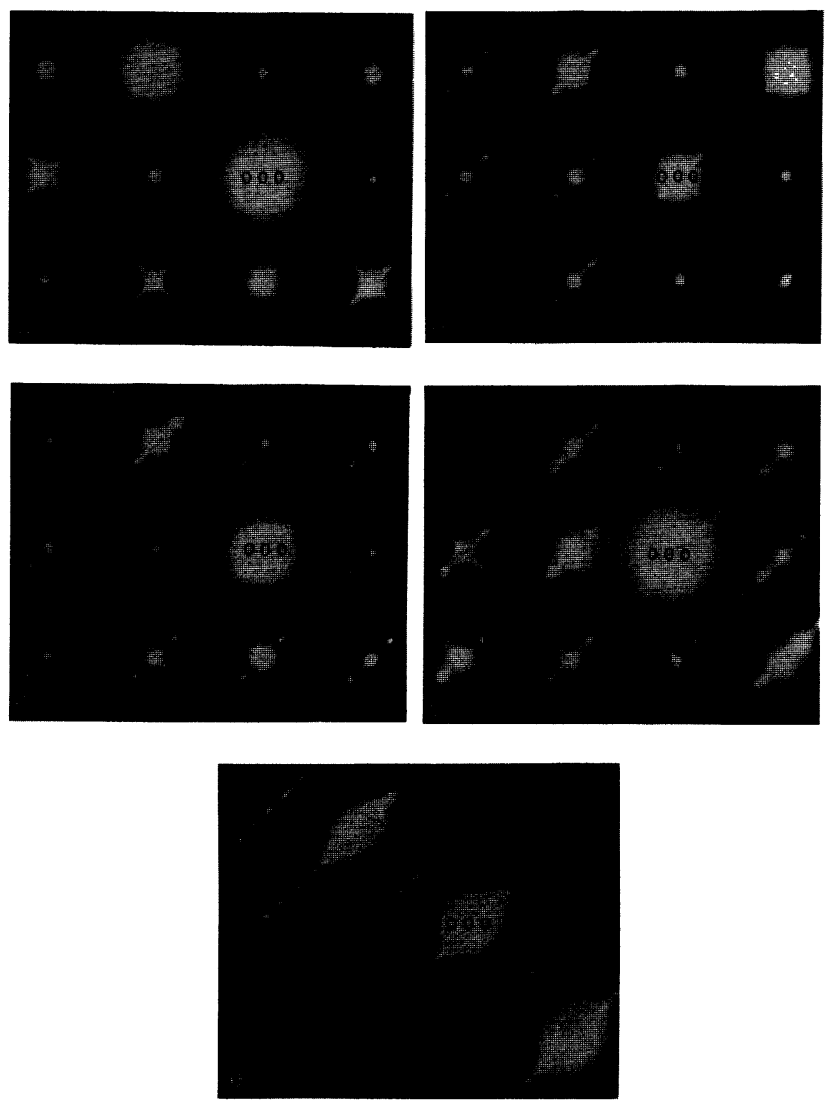

Fig. 9. - Transition de la matrice $\beta^{\prime}$ (Fig. 9a) à la martensite ou à une phase intermédiaire martensitique (Fig. $9 e$, [117]) dans $\mathrm{Ni}$ 37,5 at $\% \mathrm{Al}$ recuit 3 jours à $1150^{\circ} \mathrm{C}$ et refroidi rapidement [116].

[Transition from the $\beta^{\prime}$ phase (Fig. 9a) to the martensite or to an intermediate martensitic phase (Fig. $9 e,[117]$ ) in a $\mathrm{Ni}-37.5$ at $\% \mathrm{Al}$ annealed three days at $11^{\circ} \mathrm{C}$ and rapidly cooled.]

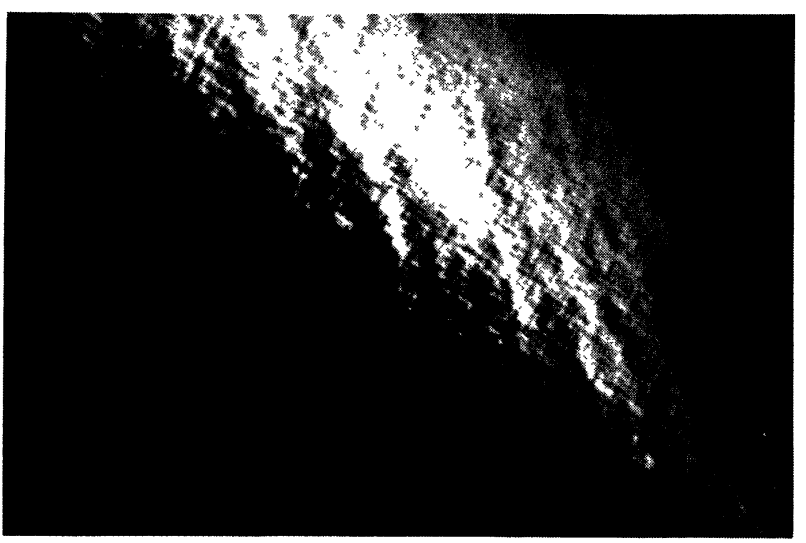

Fig. 10. - Anomalies de contraste en champ clair dans les alliages $\mathrm{Ni}-\mathrm{Al}$, correspondant à la figure $8 a$.

[Anomalies in the bright field electron micrographs of $\mathrm{Ni}-\mathrm{Al}$ alloys, corresponding to figure $8 a$.]

3. 3 LE COMPORTEMENT PRÉMARTENSITIQUE DE NiAl. - Des phénomènes intéressants ont été mis en évidence dans le composé $\mathrm{NiAl}$ : il s'agit de traînées diffuses parallèles aux directions $\langle 110\rangle^{*}([40]$, [113], Fig. 8). La nature physique de ces traînées fait l'objet d'une intéressante controverse [114], [115]. D'après Reynaud [116], elles constitueraient bien un phénomène précurseur de la martensite (Fig. 9); elles ne proviendraient donc pas de la précipitation de $\mathrm{Ni}_{2} \mathrm{Al}$ [40], [115]. D'après [114], [117], ces traînées seraient même la conséquence directe de l'instabilité du cristal par rapport au mode de phonon correspondant à la constante de cisaillement $C^{\prime}$ (mode transverse acoustique de centre de zone), mais d'autres travaux sont nécessaires avant de pouvoir conclure sur ce sujet en pleine controverse... Des phénomènes analogues ont été observés dans d'autres systèmes, comme par exemple $\mathrm{Fe}_{3} \mathrm{Pt}$ [118]. Enfin, nous présentons à la figure 10 la micrographie électronique en champ clair correspondant au diagramme de la figure $8 a$.

4. Conclusions. - La microscopie et la diffraction électroniques sont bien adaptées à l'étude des phénomènes prémonitoires des transitions de phases dans les alliages.

Dans le cas des solutions solides substitutionnelles, la démixtion (l'ordre à longue distance) est à la solution solide désordonnée ce qu'est le ferromagnétisme (l'antiferromagnétisme) au paramagnétisme. On peut avoir des transitions du second ordre (décomposition spinodale d'un alliage symétrique, transitions ordredésordre non frustrantes) et des transitions du premier ordre (préprécipitation dans les solutions solides terminales, transitions ordre-désordre frustrantes). La technique des ondes de concentration permet de souligner les analogies profondes entre les transitions de phases substitutionnelles et les transitions de phases avec déplacement (modes de vibration) ou les transitions magnétiques (ondes de spin).

Dans le cas des composés $\beta^{\prime}$ de Hume-Rothery, on observe de nombreuses anomalies dans les diagrammes et dans les micrographies électroniques, que l'on a réunies sous la dénomination de comportement prémartensitique. En fait, certains phénomènes (comme les préprécipités oméga) correspondent plutôt à une préprécipitation de phases intermédiaires entre la matrice c.c. (ou ses surstructures) et les phases voisines, dans le régime des températures bainitiques. On doit donc étendre les notions de préprécipitation et de zones G. P. aux composés $\beta^{\prime}$. La situation est ici toutefois plus complexe que dans le cas des solutions solides terminales, du fait de la position du domaine $\beta^{\prime}$ près du centre du diagramme de phases : on peut observer des phénomènes prémonitoires des transitions vers les phases situées à droite et à gauche du domaine $\beta^{\prime}$. D'autres phénomènes comme les traînées diffuses dans $\mathrm{NiAl}$ semblent par contre constituer un véritable comportement prémartensitique.

Les nombreuses hétérogénéités des composés $\beta^{\prime}$ (sans compter les hétérogénéités d'origine magnétique [119] ou celles dues à une éventuelle décomposition spinodale de certains composés [60]) pourraient 
jouer un rôle dans la transformation martensitique, par l'intermédiaire de modes de vibration localisés qui pourraient devenir mous et se condenser, comme les modes normaux. La notion importante de mode mou en général, et de mode mou localisé en particulier, permet ainsi de passer de manière continue de phénomènes prémonitoires dynamiques à des phénomènes statiques.
Remerciements. - L'auteur est heureux de remercier Mlle Fourdeux A., MM. de Ridder R., Thomas G., Lesbats P. et Hausch G., qui ont aimablement mis à sa disposition les diagrammes des figures $2,4,6$ et 7 .

\section{Bibliographie}

[1] MaRcinKowsKi, M. J., In Electron Microscopy and strength of crystals, Ed. Thomas and Washburn (Wiley New York) $1963,333$.

[2] Van Tendeloo, G. and Amelincks, S., In Electron Microscopy in Materials Science, Part III, Ettore Majorana, Commission of the European Communities, EUR 5515e (1975), 773.

[3] Van Landuyt, J., J. Physique Colloq. C7 38 (1977) 5.

[4] Kubo, R., Some Aspects of the Statistical-Mechanical Theory of irreversible Processes, Lectures in Theoretical Physics, vol. 1, E. Brittin \& Dunham (Interscience Publ., New York) 1959.

[5] Giraud-Héraud, F., Mem. Sci. Rev. Metall. 71 (1974) 37.

[6] Cook, H. E., Sulzawa, M., Kajitani, T. and Rivaud, L., J. Physique Colloq. C7 38 (1977) 430.

[7] Iveronova, V. I. and Katsnelson, A. A., In Order-Disorder Transformations in Alloys, Ed. Warlimont H. (SpringerVerlag) 1974, 306.

[8] Khachaturyan, A. G., Ordering in Substitutional and Interstitial Solid Solutions, Progr. Mater. Sci., Ed. Chalmers B., Christian, J. W. and Massalski, T. B. 22 (1978) 1.

[9] Swann, P. R., Duff, W. R. and Fisher, R. M., Metall. Trans. 3 (1972) 409.

[10] Morgand, P., Metall. Trans. 1 (1970) 2331.

[11] Morgand, P. et GuUrasevic, J. M., C. R. Hebd. Séan. Acad. Sci. 265C (1967) 448.

[12] Als-Nielsen, J., Phys. Rev. 185 (1969) 664.

[13] Norwell, J. C. and Als-Nelsen, J., Phys. Rev. B 2 (1970) 277.

[14] Als-Nielsen, J. and Dietrich, O. W., Phys. Rev. 153 (1967) 717.

[15] Walker, C. B. and Keating, D. T., Phys. Rev. 130 (1963) 1726.

[16] Salamon, M. B. and Lederman, F. L., Phys. Rev., B 10 (1974) 4492.

[17] MOSER Von H., Phys. Z. 21 (1936) 737.

[18] Orehotsky, J. and SCHröDer, K., J. Phys. F 4 (1974) 196.

[19] Guttman, L. and Schnyders, H. C., Phys. Rev. Lett. 22 (1969) 520.

[20] Gutrman, L., Schnyders, H. C. and Arai, G. J., Phys. Rev. Lett. 22 (1969) 517.

[21] Toulouse, G. et Vannimenus, J., La Recherche 83 (1977) 980.

[22] Sato, H., Toth, R. S. and Honjo, G., J. Phys. Chem. Solids 28 (1967) 137.

[23] Moss, S. C., Phys. Rev. Lett. 22 (1969) 1108.

[24] Moss, S. C. and WalkeR, R. H., J. Appl. Crystallogr. 8 (1975) 96.

[25] Hashimoto, S. and Ogawa, S., J. Phys. Soc. Japan 29 (1970) 710.

[26] Cowley, J. M., Acta Crystallogr. A 29 (1973) 537.

[27] Chevalier, J. P. and Stobbs, W. M., Acta Metall. 27 (1979) 285.

[28] Ohshima, K. and Watanabe, D., Acta Crystallogr. A 29 (1973) 520.

[29] Gaudig, W. and Warlimont, H., Acta Metall. 26 (1978) 709.

[30] Chevalier, J. P. and Stobbs, W. M., Acta Metall. 24 (1976) 535.

[31] Das, S. K., OKamoto, P. R., Fisher, P. M. J. and Thomas, G., Acta Metall. 21 (1973) 913.
[32] DAs, S. K., Ph. D. Thesis, Berkeley, LBL-176 (1971).

[33] Das, S. K. and Thomas, G., In Order-Disorder Transformations in Alloys, Ed. Warlimont H. (Springer-Verlag) 1974 332.

[34] Sauvage, M. and ParthÉ, E., Acta Crystallogr. A 30 (1974) 239.

[35] De Ridder, R., Van Tendeloo, G., Van Dick, D. and Amelinckx, S., J. Physique Colloq. C7 38 (1977) 178.

[36] De Ridder, R., Van Tendeloo, G. and Amelinckx, S., Acta Crystallogr. A 32 (1976) 216.

[37] De Ridder, R., Van Tendeloo, G., Van Dyck, D. and AMElINCKx, S., Phys. Status Solidi a 38 (1976) 663.

[38] De Ridder, R., Van Dyck, D., Van Tendeloo, G. and Amelinckx, S., Phys. Status Solidi a 40 (1977) 669.

[39] Van Dyck, D., De Ridder, R., Van Tendeloo, G. and Amelinckx, S., Phys. Status Solidi a 43 (1977) 541.

[40] Reynaud, F., J. Appl. Crystallogr. 9 (1976) 263.

[41] Guinier, A., Solid State Phys., Ed. F. Seitz and D. Turnbull vol. 9 (1959), 293.

[42] Hirsch, P. B., Howie, A., Nicholson, R. B., Pashley, D. W. and Whelan, M. J., Electron Microscopy of thin Crystals (Butterworths) 1965, 320.

[43] Phillips, V. A., Acta Metall. 21 (1973) 219.

[44] Phillips, V. A., Acta Metall. 23 (1975) 751.

[45] Phillips, V. A. and Tanner, L. E., Acta Metall. 21 (1973) 441.

[46] GuYot, P., J. Microsc. 98 (1973) 180.

[47] Yoshida, H., Cockayne, D. J. H. and Whelan, M. J., Philos. Mag. 34 (1976) 89.

[48] Yoshida, H., Cockayne, D. J. H. and Whelan, M. J., Proc. 8th Int. Congr. Electr. Microsc., Canberra, vol. 1 (1974) 430

[49] Yoshida, H., Hashimoto, H. and Yokota, Y., 9th Int. Congr. Electr. Microsc., Toronto, vol. 1 (1978) 306.

[50] Boyes, E. D., Gai, P. L. and Skarnulis, A. J., 9 th Int. Congr. Electr. Microsc., Toronto, vol. 1 (1978) 308.

[51] Skarnulis, A. J., J. Microsc. Spectrosc. Electr. 2 (1977) 461.

[52] CAHN, J. W., Trans. AIME 242 (1968) 166.

[53] Sinclair, R., Gronsky, R. and Thomas, G., Acta Metall. 24 (1976) 789.

[54] Laughlin, D. E., Acta Metall. 24 (1976) 53.

[55] Butler, E. P. and Thomas, G., Acta Metall. 18 (1970) 347.

[56] Reynaud, F., Lasserre, A. et Coulomb, P., Acta Metall. 23 (1975) 9.

[57] Reynaud, F., Mem. Sci. Rev. Metall. (1975) 277.

[58] Bouchard, M. and Thomas, G., Acta Metall. 23 (1975) 1485.

[59] Coulomb, P., J. Microsc. Spectr. Electr. 2 (1977) 389.

[60] Kubo, H. and Wayman, C. M., Acta Metall. 10A (1979) 633.

[61] Willaime, C., J. Microsc. Spectrosc. Electr. 2 (1977) 405.

[62] Cochran, W., Adv. Phys. 9 (1960) 387.

[63] Cochran, W., Adv. Phys. 10 (1961) 401.

[64] Fleury, P. A., Ann. Rev. Mater. Sci. 6 (1976) 157.

[65] ScotT, J. F., Rev. Mod. Phys. 46 (1974) 83.

[66] Blinc, R. and Zeks, B., Soft Modes in ferroelectrics and antiferroelectrics, North-Holland/American Elsevier (1974), Ed. Wohlfarth.

[67] Perkins, J., Scr. Metall. 8 (1974) 31.

[68] Perkins, J., Scr. Metall. 8 (1974) 975.

[69] Nagasawa, A., Phys. Lett. 45A (1973) 265. 
[70] Nakanishi, N., In Shape Memory in Alloys, Ed. J. Perkins (Plenum Press) 1975, 147

[71] Vatanayon, S. and Hehemann, R. F., In Shape Memory in Alloys, Ed. Perkins J. (Plenum Press) 1975, 115.

[72] Clapp, P. C., Phys. Status Solidi b 57 (1973) 561.

[73] Suzuki, T. and Wuttig, M., Acta Metall. 23 (1975) 1069.

[74] Delaey, L., Perkins, A. J. and Massalski, T. A., J. Mater. Sci. 7 (1972) 1197.

[75] Chandra, K. and Purdy, G. R., J. Appl. Phys. 39 (1968) 2176.

[76] Sandrock, G. D., Perkins, A. J. and Hehemann, R. F., Metall. Trans. 2 (1971) 2769.

[77] Dautovich, D. P. and Purdy, G. R., Can. Met. Quart. 4 (1965) 129.

[78] Zijlstra, S. R., Beijer, J. and Klostermann, J. A., J. Mater. Sci. 9 (1974) 145.

[79] Koskimaki, D., Marcinkowski, M. J. and Sastri, A. S., Trans. AIME 245 (1969) 1883

[80] Wang, F. E., Pickart, S. J. and Alperin, H. A., J. Appl. Phys. 43 (1972) 97.

[81] Nishiyama, Z., Morikawa, H. and ShimizU, K., Japan J. Appl. Phys. 6 (1967) 815

[82] De Bondt, M. and Deruyttère, A., Acta Metall. 15 (1967) 993.

[83] Vandermeulen, W. and Deruyttère, A., Metall. Trans. 4 (1973) 1659.

[84] Warlimont, H., 6th Int. Congr. Electr. Microsc. Kyoto (1966) 437

[85] Kubo, H., Shimizu, K. and Wayman, C. M., Metall. Trans. 8A (1977) 493.

[86] Mukherjee, K., Chandrasekaran, M. and Milillo, F., In Shape Memory Effects in Alloys, Ed. J. Perkins (Plenum Press) $1975,177$.

[87] Sato, S. and Takezawa, K., Suppl. Trans. Japan Inst. Met. 9 (1968) 925

[88] VAN PAEMEL, J., Burgerlijk Metaalkundig Ingenieur Eindwerk, Université catholique de Louvain '(1971)

[89] TakeZawa, K. and SATo, S., J. Japan Inst. Met. 37 (1973) 793.

[90] Prasetyo, A., Reynaud, F. and Warlimont, H., Acta Metall. 24 (1976) 1009.

[91] Murakami, Y., Delaey, L. and Smeesters-Dullenkopf, G. Trans. JIM 19 (1978) 317.

[92] Buron, C., Thèse d'Etat de Chimie, Université de Besançon (1977).

[93] Prasetyo, A., Reynaud, F. and Warlimont, H., Acta Metall. 24 (1976) 651.
[94] Prasetyo, A., Reynaud, F. and Warlimont, H., Proc. IV Cong. Int. Microsc. Electr. Haute Tension, Toulouse (1975) 253

[95] Corey, C. L. and TAtefF, K. M., Scr. Metall. 10 (1976) 909.

[96] Stobis, W. M., Henderson, R. J. and Crossley, A. M., INCOMAT 1979.

[97] Kubo, H., Hamabe, A. and Shimizu, K., Scr. Metall. 10 (1976) 547.

[98] Williams, J. C., De Fontaine, D. and Paton, N. E., Metall. Trans. 4 (1973) 2701.

[99] Kuan, T. S. and Sass, S. L., Philos. Mag. 36 (1977) 1473.

[100] Weber, D., Meurtin, M., Paris, D., Fourdeux, A. and Lesbats, P., J. Physique Colloq. 38 (1977) C7-332.

[101] Kirchgraber, G. and Gerold, V., J. Appl. Crystall. 11 (1978) 153 .

[102] Berner, D., Doktor Arbeit Stuttgart (1976)

[103] Van Paemel, J., Cottam, R. and Delaey, L., Z. Metallkd. 66 (1975) 453.

[104] Chandrasekaran, M., Delaey, L., Van Paemel, J. and RAPACIOLI, R., Z. Metallkd. 67 (1976) 323.

[105] Hehemann, R. F., Can. Metall. Quart. 11 (1972) 201

[106] Von Hornbogen, E., Z. Metallkd. 50 (1959) 70.

[107] KaminskiJ, E., Tech. Phys. USSR 5 (1938) 953.

[108] Mori, M., Yamada, Y. and Shirane, G., Solid State Commun. 17 (1975) 127

[109] Guenin, G., Hautecler, S., Pynn, R., Gobin, P. F. and DelaEY, L., Scr. Metall. 13 (1979) 429.

[110] Madelung, O., Festkörpertheorie III (Springer Verlag) 1973, 70.

[111] Maradudin, A. A., Solid State Phys. 18 (1966) 273.

[112] Sanchez, J. M. and De Fontaine, D., Acta Metall. 26 (1978) 1083.

[113] Lasalmonie, A., C. R. Hebd. Séan. Acad. Sci. 280C (1975) 1501 .

[114] Enami, K., Hasunuma, J., Nagasawa, A. and Nenno, S., Scr. Metall. 10 (1976) 879

[115] Lasalmonie, A., Scr. Metall. 11 (1977) 527.

[116] Reynaud, F., Scr. Metall. 11 (1977) 765.

[117] Enami, K., Nagasawa, A. and Nenno, S., Scr. Metall. 12 (1978) 223.

[118] Foos, M., Frantz, C. and Gantois, M., Scr. Metall. 12 (1978) 795

[119] KraSEveC, V., Icomat (1977), Kiev, 32. 